\title{
GORTER-MELLINK HEAT TRANGPORT IN HE-II TRANSVERSE TO TUBE BANKS AND THROUGH DUCTS OF NONUNIFORM CROSS SECTION
}

\author{
Lawrence Dresner \\ Oak Ridge National Laboratory, Oak Ridge, TN 37831, USA
}

\begin{abstract}
Heat transport in the practical range of fluxes $\left(\sim 1 \mathrm{~W} / \mathrm{cm}^{2}\right)$ obeys the nonlinear Gorter-Mellink relation $\vec{q}=-K(\nabla T)^{1 / 3}$. In this paper, we study steady-state Gorter-Mellink heat transport (1) through a plane duct of nonuniform cross section and (2) transverse to a bank of parallel tubes with adiabatic surfaces rranged in either a square or triangular lattice. Both problems are solved by the method of complementary variational principles.
\end{abstract}

\section{INTRODUCTION AND RESULTS}

Heat transport in helium-Il in the practical range of fluxes $\left(\sim 1 \mathrm{~W} / \mathrm{cm}^{2}\right)$ obeys the nonlinear equation $\vec{q}=-K(\nabla T)^{1 / 3}$, where $\vec{q}$ is the heat flux vector, $\nabla T$ is the temperalure gradient, and $K$ is a constant of proportionality [1]. Gorter and Mellink [2] accounted for this equation by adding to the two-fluid equations terms that introduce mutual friction between the superfluid and the normal fuid. Accordingly, the ieregoing equation is sometimes called by their names. When combined with the equation of continuity, $\nabla \cdot \vec{q}+S(\partial T / \partial t)=0$, the Gorter-Mellink equation leads to the nonlinear, diffusion-like equation

$$
S(\partial T / \partial t)=\nabla \cdot\left[K(\nabla T)^{1 / 9}\right]
$$

where $S$ is the heat capacity per unit volume. It is convenient to refer to tive heat transport process that Eq. (1) describes as Gorter-Mellink diffusion, even though internal convection of the two fluids, not diffusion, is responsible for the transport of the heat.

In this paper, we study steady-state Gorter-Mellink diffusion in two situations: (1) through a plane duct of nonuniform cross section and (2) transverse to a bank of parallel tubes, whose surufaces are adiabatic, arranged in either a square or a triangular lattice. In either case, we imagine a pair of parallel planes a distance $\Delta x$ apart. One plane is maintained at the temperature $T_{2}$, and the other at the lower temperature $T_{1}$. In case 1 , the plane duct is formed by these two end planes and the adiabatic surfaces $y=\zeta_{2}(x)$ and $y=Y_{1}(x)$, with $Y_{2}>Y_{1}$. In case 2, the parallel isotlierms include a portion of the lattice of tubes (see the inserts in Fig. 1). The distance $\Delta x \gg 2 L ; 2 L$ is the pitch (center-to-center distance) of the tube lattice. In either case, we wish to know the total heat flow between the isothermal end planes. In case 2, we express the heat flux as a fraction $f$ of the heat flux $\left.K\left(T_{2}-T_{1}\right) / \Delta x\right]^{1 / 3}$ that would flow in the absence of the tubes. The temperature dependence of $K$ is ignored in this paper.

Under the condition $\Delta x \gg 2 L$, the fraction $f$ does not depend on the angle the planes make with the symmetry axes of the tube lattices. This conclusion is a consequence of the high geometric symmetry of the square and triangular lattices and can be proved by group-theoretic means.

Figure 1 shows the fraction $f$ for both lattices versus the diameter-to-pitch ratio $\rho$. The numerical values of $f$ were obtained from a pair of complementary variational principles $[3.4\}$, one of which gives upper bounds to the exact values and the other of which gives lower bounds. 
Twe curves are shown for each lattice, one an upper bound and the other a lower bound. For the square lattice, the percent difference between these bounds has a maximum of about $10 \%$ in the neighborhood of $\rho=0.3$. For reasons that will be given later, the upper bounds are expected to have second-order (variational) accuracy while the lower bounds are not. The function $f=(1-\rho)(1+2 \rho) /(1+\rho)$ fits the two upper bounds well (within $4 \%$ for $\rho<0.85)$ and is recommended for design purposes.

\section{COMPLEMENTARY VARIATIONAL PRINCIPLES}

The following complementary variational principles are applicable to heat transport through an irregularly shaped duct, whose (plane parallel) ends are isothermal surfaces and whose sides are adiabatic walls. Reference [4] gives a thorough description of how to construct a pair of variational principles; therfore, we simply state the variational principles for our problem. Let

$$
\begin{gathered}
A_{+}=\int_{V}|\nabla T|^{4 / 3} d V \\
A_{-}=-\frac{1}{3 K^{4}} \int_{V} q^{4} d V-\frac{4}{3 K} \int_{S} T \cdot \vec{q} \cdot \vec{d} s
\end{gathered}
$$

where $V$ is the interior volume of the channel, $S$ is its total surface, $T$, is the exact solution corresponding to the isothermal-adiabatic boundary conditions given above, $T$ is a trial function obeying the boundary conditions $T=T_{2}$ and $T=T_{1}$ on the isothermal surfaces, and $\vec{q}$ is a divergenceless trial vector. Then, it can be shown that

$$
A_{+} \geq A_{.}=Q\left(T_{2}-T_{1}\right) / K \geq A_{-}
$$

with equality holding when $T=T$. and $\vec{q}=-K(\nabla T .)^{1 / 3}$. Here $Q$ is the total heat flow through the duct.

\section{APPLICATION TO THE IRREGULARLY SHAPED DUCT}

We take the isothermal end surfaces to be perpendicular to the $x$-axis and located at $x=0$ and $x=D$. Suppose we choose our trial iunction to be a function of $x$ only [i.e., $T=T(x)$ ). To satisfy the boundary conditions on the isothermal surfaces we must have $T(0)=T_{2}$ and $T(D)=T_{1}$. Then,

$$
A_{+}=\int_{0}^{D}\left(\frac{d T}{d x}\right)^{4 / 3}\left(Y_{2}-Y_{1}\right) d x
$$

We choose the dependence of $T$ on $x$ to make $A_{+}$a minimum. Finding this dependence is a straightforward variational problem, but restrictions on the length of this article preclude giving the details of its solution here. Those details can be found in Ref. [5]. By using this best $T(x)$, we find

$$
Q / K\left(T_{2}-T_{1}\right)^{1 / 3} \leq\left[\int_{0}^{D} d x /\left(Y_{2}-Y_{1}\right)^{3}\right]^{-1 / 3}
$$

where $Q$ is the total heat flow through the duct (per unit length in the direction perpendicular to the $\mathrm{x}$ and $\mathrm{y}$ axes). Now let us evaluate $A_{-}$. The trial vector $\vec{q}$ must be divergenceless, so we introduce a stream function $\psi: q_{x}=\partial \psi / \partial y, q_{y}=-\partial \psi / \partial x$. Then,

$$
A_{-}=-\frac{1}{3 K^{4}} \int_{V}(\nabla \psi)^{4} d V-\frac{4}{3 K} \int_{S} T_{*}\left[d S_{x}(\partial \psi / \partial y)-d S_{y}(\partial \psi / \partial x)\right]
$$

Equation (6) contains $T_{*}$ on the right-hand side, and $T_{*}$ is known only on the isothermal surfaces (from the boundary conditions). To eliminate this difficulty, let us restrict ourselves to trial stream functions $\psi$ for which the adiabatic walls $Y_{2}(x)$ and $Y_{1}(x)$ are level surfaces (i.e., surfaces on which $\psi$ is constant $)$. Then the trial vector $\vec{q}=(\partial \psi / \partial y,-\partial \psi / \partial x)$ is parallel to the adiabatic walls at the walls, and thus they give no contribution to the surface integral. The 
surface integral then becomes $4\left(T_{2}-T_{1}\right)\left(\psi_{2}-\psi_{1}\right) / 3 K$ where $\psi_{2}$ and $\psi_{1}$ are the values of $\psi$ on the level surfaces $Y_{2}$ and $Y_{1}$, respectively. If our trial functions are to include the exact solution, we must make

$$
\psi_{2}-\psi_{1}=\int_{r_{1}}^{Y_{2}}(\partial \psi \cdot / \partial y) d y=\int_{Y_{1}}^{r_{2}} q_{* x} d y=Q
$$

With this restriction, Eqs. (6) and (3) become

$$
Q\left(T_{2}-T_{1}\right) / K \leq \int_{V}(\nabla \psi)^{4} d V / K^{4}
$$

with, say, $\psi_{1}=0$ and $\psi_{2}=Q$ on $Y_{1}$ and $Y_{2}$, respectively. Despite of the direction of the inequality in Eq. (8), it will supply a lower limit to $Q$ because $\psi$ is proportional to $Q$, cwing to the boundary conditions just mentioned.

Convenient level surfaces for the trial function $\psi$ are the surfaces $y=\lambda Y_{2}(x)+(1-\lambda) Y_{1}(x)$, $0<\lambda<1$. Choosing them as level surfaces means taking $\psi=\psi(\lambda)$. As before, we let the variational principle [Eq. (8)] dictate the best assignment of values to the as yet undetermined function $\psi(\lambda)$. The most convenient way to evaluate the integral in Eq. (8) is to introduce $x$ and $\lambda$ as new coordinates. Because these new coordinates are not Cartesian, we must calculate the components of the metric tensor to evaluate the integral. Again, space limitations preclude giving the details of this calculation, which can also be found in Ref. [5]. The result is

$$
\begin{gathered}
Q / F\left(T_{2}-T_{1}\right)^{1 / 3} \geq \int_{0}^{1}\left[\left.G(\lambda)\right|^{-1 / 3} d \lambda\right. \\
G(\lambda)=\int_{0}^{D} \frac{\left\{1+\left[\lambda \dot{Y}_{2}+(1-\lambda) \dot{Y}_{1}\right]^{2}\right\}^{2}}{\left(Y_{2}-Y_{1}\right)^{3}} d x
\end{gathered}
$$

Equations (5) and (9) thus provide inclusive bounds for the quantity $Q / K\left(T_{2}-T_{1}\right)^{1 / 3}$.

\section{THE TRAPEZUIDAL DUCT}

The function $G$ is simple to evaluate when the adiabatic surfnces are straight lines (i.e., when the duct is a trapezoid). Suppose that $\dot{Y}_{1}=0$ and $\dot{Y}_{2}=-a$. Then Eqs. (5) and (9) give

$$
\left[\int_{0}^{D} d x /\left(Y_{2}-Y_{1}\right)\right]^{-1 / 3} \geq Q / K\left(T_{2}-T_{1}\right)^{1 / 3} \geq\left[\int_{0}^{D} d x /\left(Y_{2}-Y_{1}\right)^{3}\right]^{-1 / 3} \cdot \int_{0}^{1}\left(1+\lambda^{2} a^{2}\right)^{-2 / 3} d \lambda
$$

The lambda integral in Eq. (10) gives the ratio of the lower bound to the upper bound. Table 1 lists some values of this integral.

Table 1. Values of the lambda integral in Eq. (10) for several values of the slope a

\begin{tabular}{rllllllll}
\hline$a=$ & 0.0 & 0.1 & 0.3 & 0.5 & 0.7 & 1.0 & 1.5 & 2.0 \\
Integral $=$ & 1.0000 & 0.9978 & 0.9809 & 0.9504 & 0.9116 & 0.8471 & 0.7438 & 0.6565 \\
\hline
\end{tabular}

These numerical values show that even for substantial slopes the two bracketing estimates are quite close together.

\section{UPPER BOUND FOR THE SQUARE LATTICE}

Figure 2a shows a portion of the square lattice with coordinate axes indicated. The applied temperature difference is parallel to the $x$-a.jis. Symmetry requires that the lines shown parallel to the $y$-axis be isotherms and the lines shown parallel to the $x$-axis be streamlines. Streamlines 
can be considered as adiabatic walls, so the channel whose conductance we must find is the one outlined in the bold lines. We see from the drawing that $Y_{2}=L_{s}, Y_{1}=\left(R^{2}-x^{2}\right)^{1 / 2}, 0<x<R$, and $Y_{1}=0, R<x<L_{0}$. By using these specifications in Eq. (5), we find, after rearrangement, that

$$
f=Q / K\left(T_{2}-T_{1}\right)^{1 / 3} L_{0}^{2 / 3} \leq\left[\rho \int_{0}^{\pi / 2} \cos \theta(1-\rho \cos \theta)^{-3} d \theta+1-\rho\right]^{-1 / 3} ; \rho=R / L
$$

When plotted, Eq. (11) gives the curve in Fig. 1 marked "square lattice, upper bound."

\section{LOWER BOUND FOR THE SQUARE LATTICE}

If we use in $\mathrm{Eq}$. (9) the values of $Y_{1}$ and $Y_{2}$ given in the last paragraph, we find, after rearrangement, that

$$
f \geq \int_{0}^{1} d \lambda\left\{1-\rho+\rho \int_{0}^{\pi / 2} \cos \theta \frac{\left[1+(1-\lambda)^{2} \tan ^{2} \theta\right]^{2}}{(1-\rho \cos \theta)^{3}} d \theta\right\}^{-1 / 3}
$$

Now when $\theta$ is near $\pi / 2$, the $\theta$-integrand behaves like $\tan ^{3} \theta$, which means that it diverges. As a result, Eq. (12) reduces to $f \geq 0$, which, while true, is of no use.

The source of this trouble is $\dot{Y}_{1}$ which becomes infinite at $x=R$. We can avoid this difficulty and obtain a lower bound closer to $f$ by applying Eq. (9)-not to the channel shown in Fig. 2a but to the related channel shown in Fig. $2 \mathrm{~b}$, for which $Y_{1}$ remains within modest bounds. To prove the validity of this procedure, we must show that the exact value of $f$ for the channel of Fig. 2a exceeds the exact value of $f$ for the channel of $\mathrm{Fig}_{\mathrm{g}}$. $2 \mathrm{~b}$. If $T_{\text {*a }}$ represents the true temperature distribution of the channel of Fig. 2a, then

$$
A_{* a}=\int_{V_{a}}\left|\nabla T_{* a}\right|^{4 / 3} d V>\int_{V_{b}}\left|\nabla T_{* a}\right|^{4 / 3} d V>A_{* b}
$$

The first inequality follows because the volume $V_{a}$ includes the volume $V_{b}$. The second inequality follows because $T_{* a}$ is a satisfactory trial function for $T$ in the volume $T_{b}$ (it has the correct values on the isothermal surfaces, which are the same for $V_{a}$ and $\left.V_{b}\right)$. The function $G(\lambda)$ for the volume $V_{b}$ is given by

$$
\begin{aligned}
L^{2} G(\lambda)= & \rho \int_{0}^{\arcsin \rho} \cos \theta \frac{\left[1+(1-\lambda)^{2} \tan ^{2} \theta\right]^{2}}{(1-\rho \cos \theta)^{3}} d \theta \\
& +\left[1+(1-\lambda)^{2} \frac{\rho^{2}}{1-\rho^{2}}\right]^{2} \frac{\sqrt{1-\rho^{2}}}{2 \rho}\left[\left(1-\rho \sqrt{1-\rho^{2}}\right)^{2}-1\right]
\end{aligned}
$$

When used in Eq. (9a), Eq. (14) results in the curve plotted in Fig. 1 and marked "square lattice, lower bound." Because this lower bound applies to the volume $V_{b}$ rather than the volume $V_{a}$, it no longer has variational (second-order) accuracy. It is nonetheless a rigorous lower bound.

In an entirely similar manner, the corresponding curves for the triangular lattice have been calculated for $\rho<\sqrt{3} / 2$, and they, too, are plotted in Fig. 1 .

\section{CONCLUDING REMARKS}

The most immediate application of this work is to tube-in-bath heat exchangers, for which it can be used to calculate the maximum rate of heat transfer that does not cause volume boiling in the bath. Treating the tube surfaces as adiabatic is not rigorous, but if the heat flux through a given tube is only a small fraction of the total heat flux across the tube bank, it is a reasonable approximation.

\section{DISCLAIMER}

This report was prepared as an account of work sponsored by an agency of the United States Government. Neither the United States Government nor any agency thereof, nor any of their employees, makes any warranty, express or implied, or assumes any legal liability or responsibility for the accuracy, completeness, or usefulness of any information, apparatus, product, or process disclosed, or represe is that its ust: would not infringe privately owned rights. Reference herein to any specific a mmercial product, process, or service by trade name, trademark, manufacturer, or otherwise does not necessarily constitute or imply its endorsement, recommendation, or favoring by the United States Government or any agency theresf. The views and opinions of authors expressed herein do not necessarily state or reflect those of the United States Government or any agency thereof. 


\section{ACKNOWLEDGEMENT}

Research sponsored by the Office of Fusion Energy, U.S. Department of Energy, under contract DE-AC05-840R21400 with Martin Marietta Energy Systems, Inc. Work performed in part while the author was on assignment to the Applied Superconductivity Center, University of WisconsinMadison.

\section{REFERENCES}

1 W. H. Keesom and B. F. Saris, Physica 7:241 (1940).

2 C. J. Gorter and J. H. Mellink, Physica 15:285 (1949).

3 B. Noble and M. J. Sewell, "On Dual Extremum Principles in Applied Mathematics," J. Inst. M: :.. is Appl. 8:123-93 (1972).

4 A. M. Arthurs, Complementary Variational Principles, Clarendon Press, Oxford, 1970.

5 L. Dresner, "Nonlinear Differential Equations," ORNL/TM-10655, Sects 6.9-6.11, Oak Ridge National Laboratory, Oak Ridge, Tennessee, January 1988. A vailable from National Technical Information Service, U.S. Department of Commerce, 5285 Port Royal Road, Springfield, VA 22161.
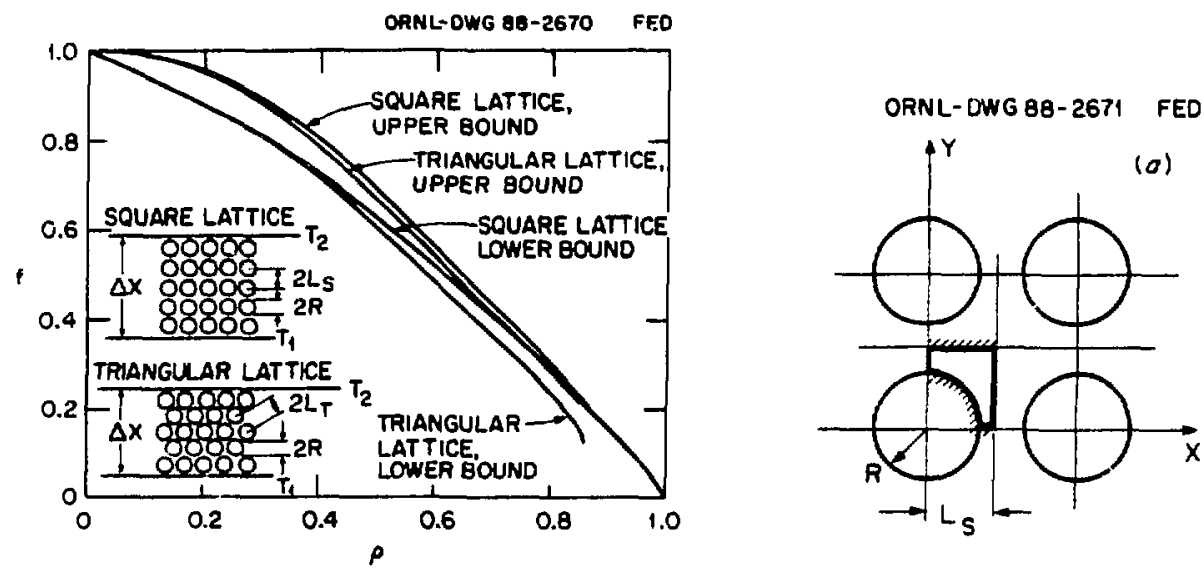

Fig. 1 Upper and lower bounds to the correction factor $f$ for the square and triangular lattices as a function of $\rho$, the diameter-lo-pitch ratio.

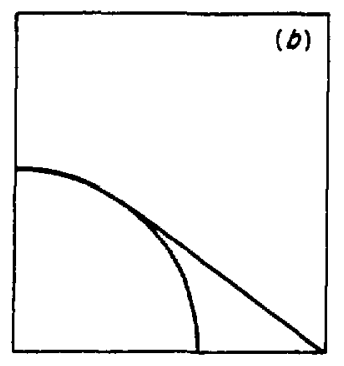

Fig. 2 Sketch showing (a) the unit channel for the square lattice and $(b)$ the effective channel for calculating a lower bound. The hatched surfaces are adiabatic. 